\title{
The tandem cycloaddition chemistry of nitroalkenes
}

\author{
Scott E. Denmark, Mark E. Schnute, Atli Thorarensen, Donald S. Middleton, \\ and Andreas Stolle \\ Department of Chemistry, University of Ilinois at Urbana-Champaign \\ Urbana, Illinois, 61801, USA
}

\begin{abstract}
Nitroalkenes have proven to be extremely versatile $4 \pi$-components in heterodiene [4+2]-cycloadditions. Our efforts in this area have been intensively methodological with focus on the reaction scope, mechanism, stereoselectivity and transformations of the cycloadducts. The three modes of tandem inter $[4+2] /$ intra $[3+2]$ cycloaddition, fused, spiro and bridged have been documented. An efficient and highly selective synthesis of (-)-hastanecine has been completed.
\end{abstract}

As part of our early investigation of the heterodiene cycloaddition reactions of nitroalkenes, we recognized the potential of the cyclic nitronic esters that result from the [4+2] process to serve as the $4 \pi$ components in a second, [3+2] dipolar, cycloaddition (1). Of the four conceivable permutations of interand intramolecularity for this coupled process, the most powerful and extensively investigated has been the tandem inter $[4+2] /$ intra [3+2] reaction (2). This permutation enjoys the advantages of (a) ease of preparation of the precursors, (b) flexibility in the electronic nature and configuration of the components, (c) high levels of relative stereocontrol, (d) the potential for absolute stereocontrol by chiral auxiliaries, (e) ease of manipulation of the cycloadducts and (f) diversity of product structure.

Under the rubric of tandem inter [4+2]/ intra [3+2] cycloadditions we have recognized three subclasses that are defined by the point of attachment of the dipolarophile to the nitronate dipole, Scheme I. In the fused mode, the dipolarophile is tethered to the $\beta$-carbon of the nitroalkene and the product is a laterally fused nitroso acetal. In the spiro mode, the dipolarophile is tethered to the $\alpha$-carbon of the nitroalkene and the product is a spiro fused nitroso acetal. Finally, in the bridged mode, the dipolarophile is tethered to the dienophile at either the $\alpha$ - or $\beta$-carbon and the product is a bridged tricyclic nitroso acetal. The final products from each of these tandem processes will vary with the nature of the dipolarophile, but in all cases the nitroso acetal is unmasked by a simple hydrogenation reaction.

\section{Scheme I}

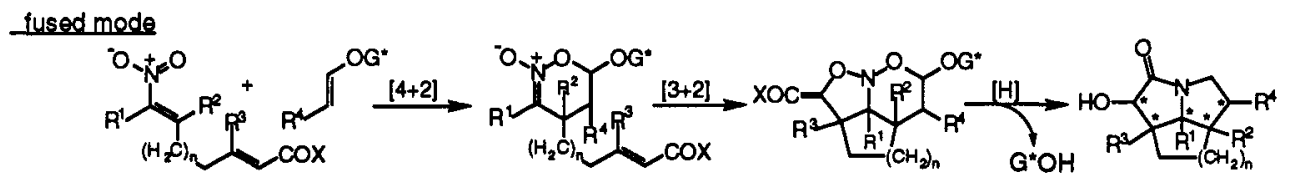

soiromode

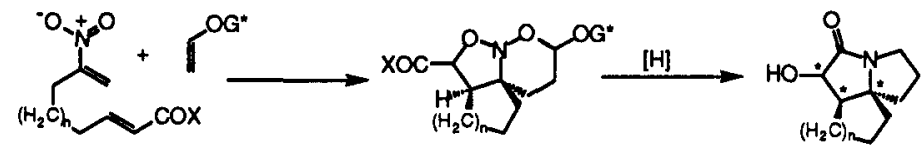

bridged mode

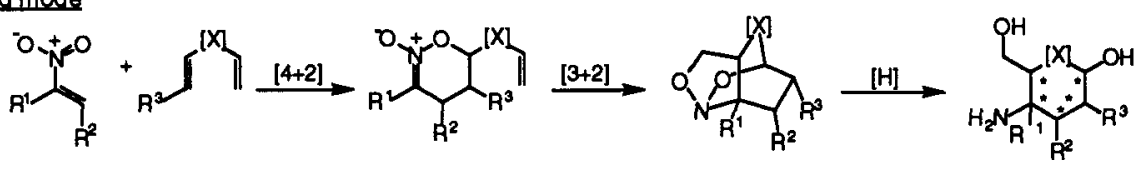


Fused Mode Cycloaddition. Chiral Vinyl Ether Dienophiles. The high degree of relative stereocontrol observed in the fused mode cycloaddition of 1 with butyl vinyl ether and the release of the vinyl ether appendage in the cleavage augured well for the success of asymmetric cycloadditions using chiral vinyl ethers. Three chiral vinyl ethers, $\mathbf{2 a}, \mathbf{2 b}$, and $\mathbf{2 c}$, derived from readily available secondary alcohols were surveyed for their ability to discriminate the enantiofaces of the nitroalkene 1 . In all cases the tandem cycloadditions proceeded smoothly in the presence of $\operatorname{Ti}(i-\mathrm{PrO}){ }_{2} \mathrm{Cl}_{2}$ to afford a mixture of anomers 3 and $3^{\prime}$ favoring the $\alpha$-oriented alkoxy group (endo mode [4+2]). The anomeric nature of these isomeric pairs was demonstrated by the independent hydrogenation of each to afford the same tricyclic lactam 4 in excellent yields along with a high recovery of the auxiliaries, Scheme II. The enantiomeric excess of 4 was determined by chiral HPLC analysis and absolute configuration was established by the method of Mosher. The extreme enantioface selectivity displayed by the different vinyl ethers has been rationalized in terms of an intrinsic preference for the endo orientation of the strans reactive conformation of 2 . The nature of the $\pi$-face shielding and the remarkable inversion of absolute configuration of 4 with the use of aluminum-based Lewis acids has been discussed in detail (3).

\section{Scheme II}

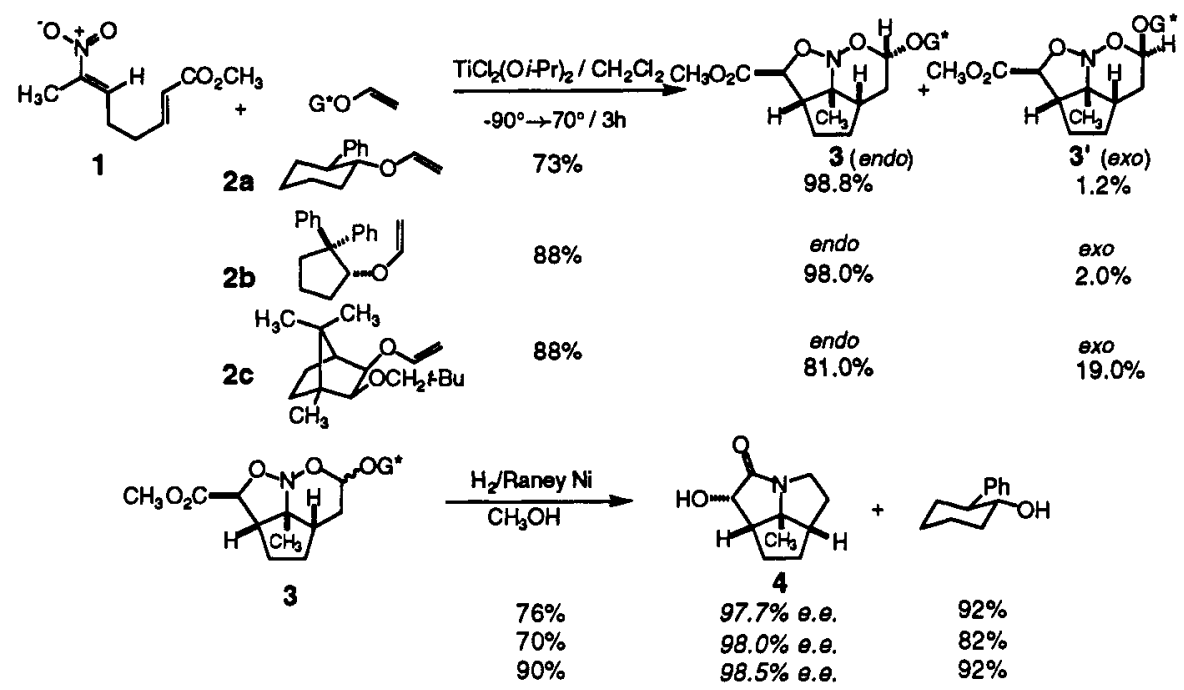

Synthesis of (-)-Hastanecine. (-)-Hastanecine is the basic portion of the pyrrolizidine alkaloid Hastacine. The structure of (-)-hastanecine was established by Culvenor in 1968 and has been synthesized several times since (4). Our objective in considering the synthesis of (-)-hastanecine was simply to illustrate the power of the tandem cycloaddition for stereocontrolled construction of substituted pyrrolidines. Careful analysis of the stereochemical relationship between the ring fusion and the hydroxymethyl substituent dictated that the [3+2] cycloaddition be intermolecular and that the dipolarophile be a maleate ester. The synthesis, Scheme III, follows simply from the stereochemical principles outlined above. Starting with 2-benzyloxynitroethylene, (5) cycloaddition with chiral vinyl ether 2 a proceed smoothly to give nitronate 5 as a single diastereomer! Treatment with dimethyl maleate in benzene afforded the nitroso acetal 6 again as a single diastereomer. From previous experience we expected the dipolarophile to react on the face opposite the benzoyloxy group and in an exo mode. Hydrogenolytic unmasking of the nitroso acetal afforded the $\alpha$-hydroxy lactam 7 whose correct relative and absolute configuration were established by X-ray analysis of the $(S)$-mandelate ester. Removal of the superfluous hydroxyl group via the phenylthionobenzoate 8 was uneventful (6). Final 
reduction/deprotection of 9 afforded (-)-hastanecine identical with an authentic sample (7). The overall yield for this six step synthesis was $21 \%$, the shortest, highest yielding and most selective to date.

Scheme III
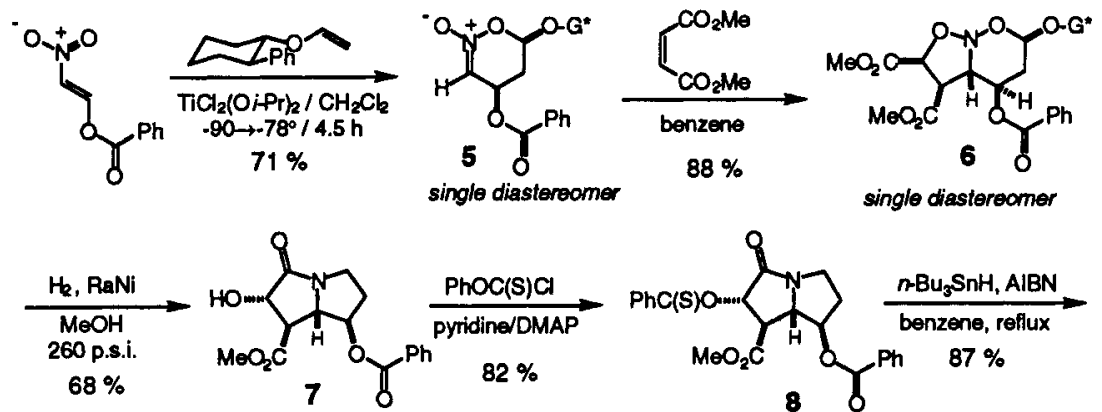
$97.7 \%$ ee (HPLC)
X-ray of (S)-mandelate
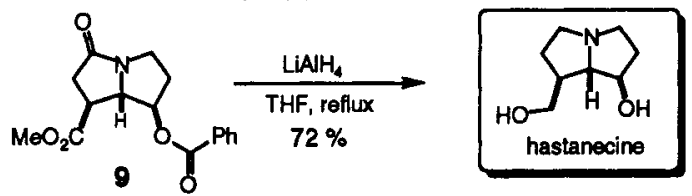

\section{$21 \%$ overall}

$[\alpha]_{D}-10.4^{\circ}(\mathrm{EtOH}) ; \mathrm{mp} 111-112^{\circ} \mathrm{C}$ Lit: $\left[\right.$ odd $-10.0^{\circ}(\mathrm{EtOH}) ; \mathrm{mp} 113-114^{\circ} \mathrm{C}$

Spiro Mode Cycloaddition. Attachment of the dipolarophile tether at the $\alpha$-position leads to nitronates that create spirocyclic nitroso acetals in the [3+2] process. This concept is illustrated in Scheme IV for the pair of $\alpha$-tethered nitroalkenes containing three and four methylene units. Both substrates 10 and 13 underwent cycloaddition readily with MAD (8) as the Lewis acid followed by brief warming to effect complete [3+2] cycloaddition. The nitroso acetals 11 and 14 were formed with unexpectedly high diastereoselectivity. It should be noted that the anomeric center is the only resident stereocenter in the nitronate and exerts a strong stereodirecting influence on the folding of the tether. Hydrogenolysis at 160 psi afforded the spiro tricyclic lactams 12 and 15 in good yield.

\section{Scheme IV}
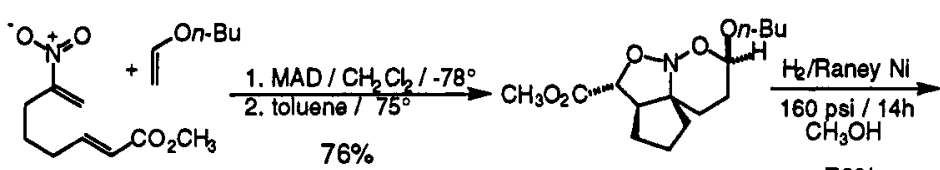<smiles>O=C1C(O)C2CCC3C2CCN13</smiles>

10

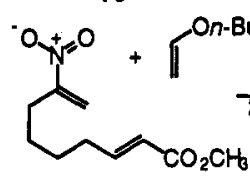

13

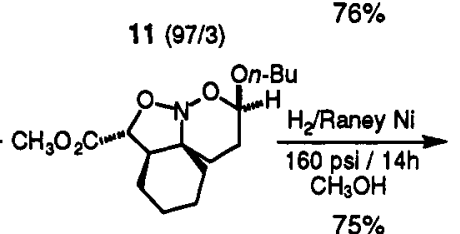

$14(96 / 4)$

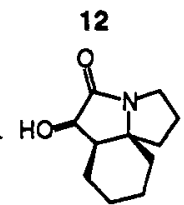

To effect absolute stereocontrol we assayed the chiral vinyl ether $\mathbf{2 a}$ in cycloadditions with $\mathbf{1 0}$ and 13. While MAD gave disappointing results ( $2 \%$ e.e.), MAPH lead to excellent yields of the cycloadducts 16 and 17 with high diastereoselectivity. Hydrogenolytic cleavage of the nitroso acetals afforded 12 and 15 in 83 and $89 \%$ e.e. respectively with excellent recovery of the chiral alcohol, Scheme V.

Bridged Mode Cycloaddition. If one focuses on the nitronate rather than the nitroalkene for opportunities to attach the dipolarophile, then two other positions become obvious, $C(5)$ and $C(6)$ of the nitronate ring. Attachment of the tether at the $C(6)$ position requires the use of a 1,4-diene. In 


\section{Scheme V}

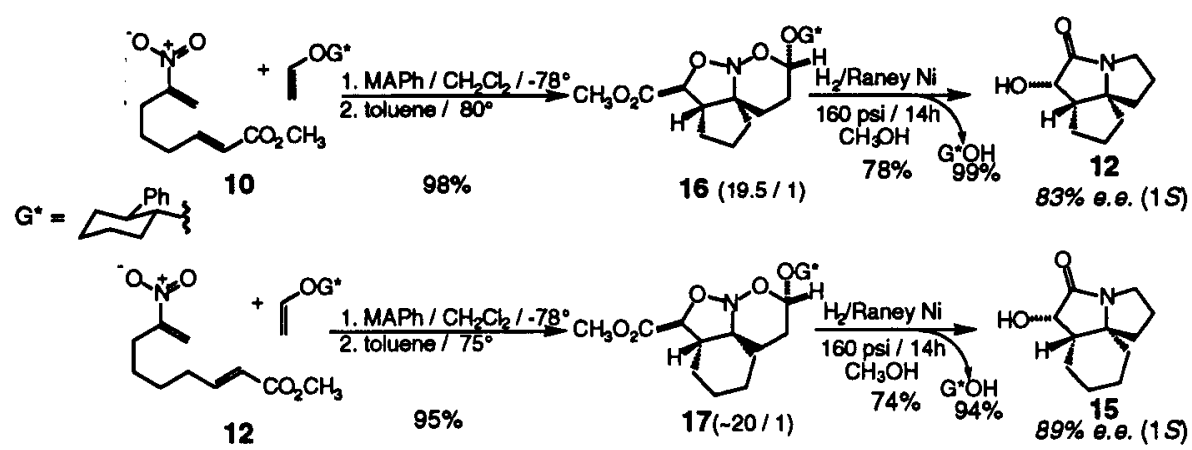

preliminary experiments, we have found that nitrostyrene 18 reacted readily with 3,3-dimethyl-1,4pentadiene in the presence of $\mathrm{SnCl}_{4}$ to produce nitronate 19 in excellent yield as a single diastereomer arising from exo mode cycloaddition, Scheme VI. Heating 19 effected [3+2] cycloaddition to produce bridged bicyclic lactone 20 in $96 \%$ yield. Hydrogenolytic cleavage followed by exhaustive acetylation afforded the polyfunctional cyclohexane derivative 21 whose stereostructure was assured by X-ray analysis. Many other variations on this theme are currently being explored.

\section{Scheme VI}

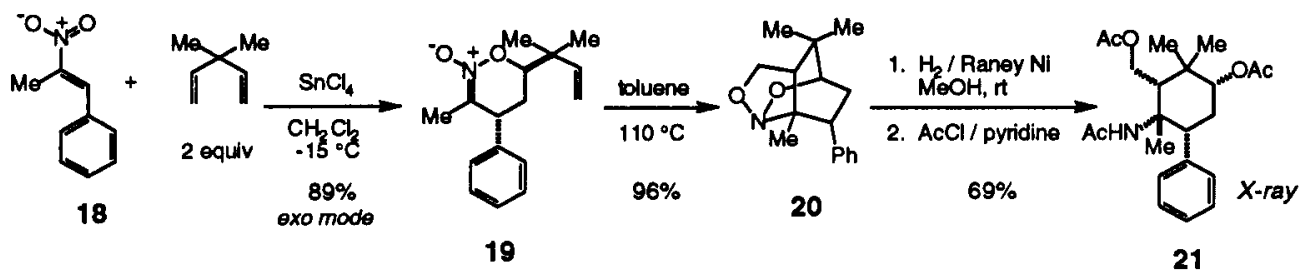

Acknowledgment. We are grateful to the National Institutes of Health (GM 30938) for support of this research. M.E.S. thanks the Organic Division of the ACS for a graduate fellowship. D.S.M. thanks Merck, Sharp and Dohme for a postdoctoral fellowship. A.S. thanks the Alexander von Humbodlt foundation for a Feodor Lynen fellowship.

\section{References}

(1) S.E. Denmark, C.B.W. Senanayake and G.-D. Ho. Tetrahedron 46, 4857 (1990).

(2) S.E. Denmark, M. E. Schnute, C.B.W. Senanayake, Y.-C. Moon and D.S. Middleton Proceedings of the 5th International Kyoto Conference on Organic Chemistry; Y. Ohshiro and Z.-I. Yoshida, Eds.; Kodansha Press, pp. 215 (1992).

(3) S.E. Denmark, M.E. Schnute and C.B.W. Senanayake. J. Org. Chem. 58, 1859 (1993).

(4) For a recent compilation of syntheses see: J. Mulzer and M. Sharp. Synthesis 615 (1993).

(5) G.A. Kraus, J. Thurston, P.J. Thomas, R.A. Jacobsen and Y. Su. Tetrahedron Lett. 29, 1879 (1988)

(6) D.H.R. Barton and S.Z. Zard. Pure Appl. Chem. 58, 675 (1986)

(7) We are grateful to Prof. C.J. Culvenor and Dr. N. Anderton for a gift of synthetic (+)hastanecine.

(8) (a) K. Maruoka, T. Itoh, H. Yamamoto. J. Am. Chem. Soc. 107, 4573 (1985). (b) K. Nonoshita, H. Banno, K. Maruoka, H. Yamamoto. J. Am. Chem. Soc. 112, 316 (1990). 